\title{
Salivary Duct Carcinoma
}

National Cancer Institute

\section{Source}

National Cancer Institute. Salivary Duct Carcinoma. NCI Thesaurus. Code C5904.

An aggressive, high grade adenocarcinoma that arises from the salivary glands. It usually affects elderly males and presents as a rapidly enlarging mass. It metastasizes to regional lymph nodes and distant anatomic sites. 